\title{
Distribution des sédiments sur la marge du golfe de Tehuantepec (Pacifique Oriental). Exemple d'interaction tectonique-eustatisme
}

El Hassane BOUMAgGARD a, Jacques GAYET b, Claude BOBIER a, Maria-Luisa MACHAIN-CASTILLO ${ }^{c}$, Eduardo AGUAYO-CAMARGO ${ }^{c}$

a Département de Géologie et Océanographie, URA CNRS 197, Université Bordeaux I, Avenue des Facultés, 33405 Talence cedex, France.

b Laboratoire CIBAMAR (Cinématique des Bassins et Marges), Université Bordeaux I, Avenue des Facultés, 33405 Talence cedex, France.

${ }^{c}$ Laboratorio de Geologia Marina, Instituto de Ciencias del Mar y de Limnologia, Universidad Nacional Autonoma de México, A P 70-157, México 04510, DF Mexique.

Reçu le 25/02/97, révisé le 11/06/97, accepté le 17/06/97.

\section{RÉSUMÉ}

Lors de la mission PROGEMA I conduite dans le golfe de Tehuantepec, des profils $3,5 \mathrm{kHz}$, de nombreux prélèvements de sédiments, des mesures hydrologiques et d'oxygène minimum jusqu'à une profondeur de $2250 \mathrm{~m}$, ont été rêalisés. Leurs études fournissent des données nouvelles pour la connaissance de cette marge active.

Ces données montrent que la distribution des dépôts du Quaternaire à ce niveau de la marge subit un double forçage tectonique et un forçage eustatique.

Le premier impact de la tectonique est lié à l'existence, dans l'isthme de Tehuantepec, de couloirs tectoniques Nord-Sud dans lesquels la circulation atmosphérique engendre des vents appelés "Tehuanos ». En interférant avec la circulation générale, ces vents répartissent les apports terrigènes selon deux provinces physiographiques et minéralogiques distinctes de part et d'autre de leur axe:

- une province sud-orientale (plaque Caraibe), alimentée depuis le Massif de Chiapas et parcourue par les masses d'eau tropicales et subtropicales véhiculées par le courant du Costa-Rica;

- une province nord-occidentale (plaque Nord Américaine), alimentée depuis la Sierra de Oaxaca et atteinte par le courant de Californie.

Le second effet de la tectonique est dû à la présence:

- de hauts-fonds sur lesquels se concentrent les produits d'une phosphatogénèse actuelle associée aux upwellings forcés par l'action des «Tehuanos »;

- de dépressions d'origine tectonique qui constituent des pièges à sédiments ;

- de nombreux canyons guidés par des accidents tectoniques qui drainent de faibles écoulements turbiditiques et canalisent les courants d'upwelling amenant sur la plate-forme des diatomées australes.

L'influence de l'eustatisme se traduit, sur la plate-forme par la présence à $-40 \mathrm{~m}$, $-75 \mathrm{~m}$ et $-130 \mathrm{~m}$, de trois niveaux de stationnement marin que l'on peut lier au niveau marin würmien et à la remontée postglaciaire. @ Elsevier, Paris. 


\section{ABSTRACT}

Distribution of sediments on the margin of the Gulf of Tehuantepec (East Pacific). Example of tectonic-eustatic interaction.

During the PROGEMA I cruise which took place on the south-western Pacific coast of Mexico (between $14^{\circ}$ and $16^{\circ} \mathrm{N}, 92^{\circ}$ and $96^{\circ} \mathrm{W}$ ), $3.5 \mathrm{kHz}$ echosounding profiles, surface samples and cores as well as hydrological and oxygen minimum measurements down to $2250 \mathrm{~m}$ water depth were made in the Gulf of Tehuantepec. Chemical, sedimentological, mineralogical, $X$-ray and BEM analyses of waters, core and surface samples provided new data which permit a better knowledge of the Gulf of Tehuantepec margin, where tectonic activity is due to an active subduction.

On the shelf of the gulf, the distribution pattern of quaternary deposits is affected by a double tectonic forcing and by eustatic oscillations of the sea level.

The first tectonic control is linked to the existence of north-south tectonic troughs in the Tehuantepec Isthmus, in which the atmospheric circulation between the Gulfs of Mexico and Tehuantepec is accelerated by the Venturi effect. The resulting winds, called "Tehuanos", play an important role in the forcing of surface circulation in the Gulf of Tehuantepec, inducing a deviation of the general circulation and thus forcing the distribution pattern of terrigenous sediment supply according to two physiographically and mineralogically distinct provinces, inherited from the past tectonic evolution, which are separated by the Tehuantepec axis.

- A southeastern province related to the Caribbean Plate and fed by: (1) the rivers draining the Chiapas Massif; and (2) the Costa-Rica Current driving tropical and subtropical waters from Central America.

- A northwestern province part of the North American Plate and fed by: (1) the Rio Tehuantepec which drains the Sierra de Oaxaca; and (2) the Californian Current.

The second tectonic control is linked to the tectonic structure of the margin influenced by:

- shallow banks where the products of a present-day phosphatogenesis are concentrated because they are swept by upwellings. The latter are reinforced because the banks are situated south of the wind corridor;

- tectonic depressions acting as sediment traps;

- numerous canyons cutting the slope along brittle accidents, driving weak turbidity currents but which play an important role in the forcing of upwelling currents. Antarctic diatoms are found which are conveyed by the deep circulation from the South-Eastern Pacific and provide evidence that Antarctic Cold Water masses (AABW) rise towards the shelf.

The influence of eustatism is attested by the presence on the shelf of the Gulf of Tehuantepec of three paleoshorelines at 40,75 and $130 \mathrm{~m}$ water depth, linked to the Würmian sea level and to the postglacial sea level rise as on many passive margins. (C) Elsevier, Paris.

Oceanologica Acta, 1998, 21, 1, 21-31.

\section{INTRODUCTION}

L'étude présentée concerne le Golfe de Tehuantepec (Fig. 1), ouvert sur le Pacifique entre $14^{\circ}$ et $16^{\circ}$ de latitude Nord et de $92^{\circ}$ à $96^{\circ}$ de longitude Ouest. Ce golfe est situé à l'aplomb du plan de subduction active à l'origine de la fosse méso-américaine. Il occupe une position clé à la convergence de trois plaques: Nord-américaine, Cocos et Caraïbes. Le cadre géologique du Golfe de Tehuantepec est de ce fait complexe (Sanchez-Barreda, 1981; Carfantan, 1986; Aguayo-Camargo et Marin, 1987; Boumaggard, 1994). L'isthme de Tehuantepec est traversé de dépressions d'origine tectonique (Carfantan, 1986) qui canalisent les circulations atmosphériques entre les golfes du Mexique et de Tehuantepec, induisant l'apparition de vents nord-sud, les «Tehuanos» (Hurd, 1929; Roden, 1961). Ces vents violents interfêrent, sur la plate-forme continentale, avec la circulation océanique générale, elle-même influencée par le cadre morpho-tectonique. La tectonique intervient ainsi, à divers niveaux pour forcer la circulation de surface, les courants d'upwelling et la distribution des dépôts sédimentaires.

Le présent travail vise à préciser le rôle de ce contrôle tectonique dans la distribution des sédiments quaternaires récents. Il intègre les résultats du traitement des données recueillies lors de la mission PROGEMA I (analyses sédimentologiques et minéralogiques effectuées sur 25 bennes et 64 carottes; examen de $200 \mathrm{~km}$ de profils d'écho- 


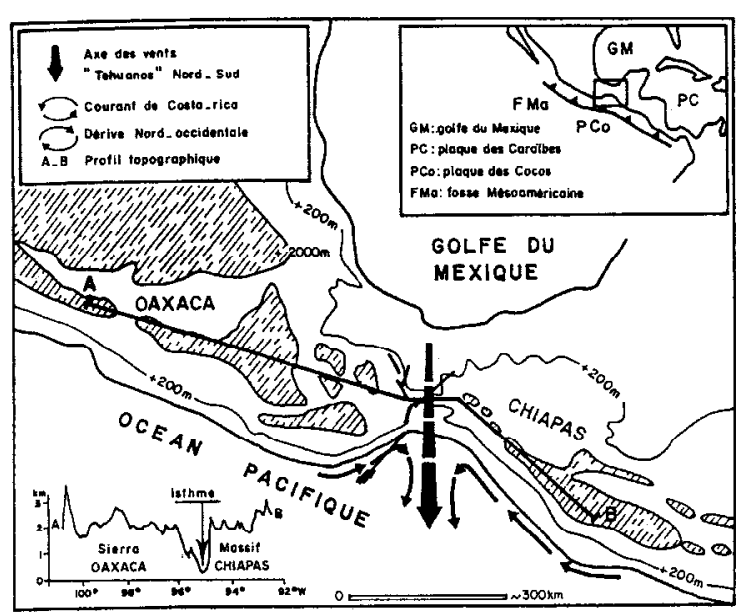

Figure 1

Contexte régional, configuration tectonique de l'isthme (failles principales) et circulation de surface dans le golfe de Tehuantepec.

Regional context, tectonic configuration of the isthmus (main faults) and surface circulation in the Gulf of Tehuantepec.

sondeurs $3,5 \mathrm{kHz}$ ) et ceux de l'analyse morphostructurale (Boumaggard et al., 1993; Boumaggard, 1994) d'une carte bathymétrique à $1 / 200000$ (INEGI 1979).

\section{CARACTÉRISTIQUES PHYSIOGRAPHIQUES \\ DE LA MARGE ET FORÇAGE TECTONIQUE \\ DE L'HYDROLOGIE ET DES FLUX DE MATIËRE}

\section{Morphostructure de l'arrière-pays}

Cet isthme cst à la jonction de deux provinces structurales (Fig. 1). A l'Ouest, atteignant $2000 \mathrm{~m}$ d'altitude, un socle cristallophyllien traversé d'intrusions magmatiques: la Sierra de Oaxaca orientée WNW-ESE. Sur sa marge Pacifique, ce bloc pratiquement dépourvu de plaine côtière et de plateau continental est caractérisé par la réduction et même l'absence de prisme de haut niveau marin.

A l'Est, une chaîne dépassant $2000 \mathrm{~m}$ d'altitude, la Sierra de Chiapas orientée NW-SE, correspondant à des formations intrusives parallèles à la côte dont elle est séparée par une vaste plaine côtière témoignant de l'existence d'un prisme de haut niveau marin.

Ces provinces présentent des structures plicatives et cassantes (Fig. 1, 2c) selon deux directions dominantes qui correspondraient à plusieurs phases de déformation (Delgado-Argote et Carballido-Sanchez, 1991).

Les plus anciennes, affectant principalement les terrains cristallins ou cristallophylliens et les séries sédimentaires anté-cénozoïques, donnent des structures NW-SE à N-S à l'origine de la formation de couloirs $\mathrm{N}-\mathrm{S}$ qui séparent les deux provinces structurales (Fig. 1) et constituent une zone de circulation facile et courte entre les golfes du Mexique et de Tehuantepec.

La plus récente est responsable de structures cassantes, d'orientation NE-SW, qui témoignent de l'influence domi-
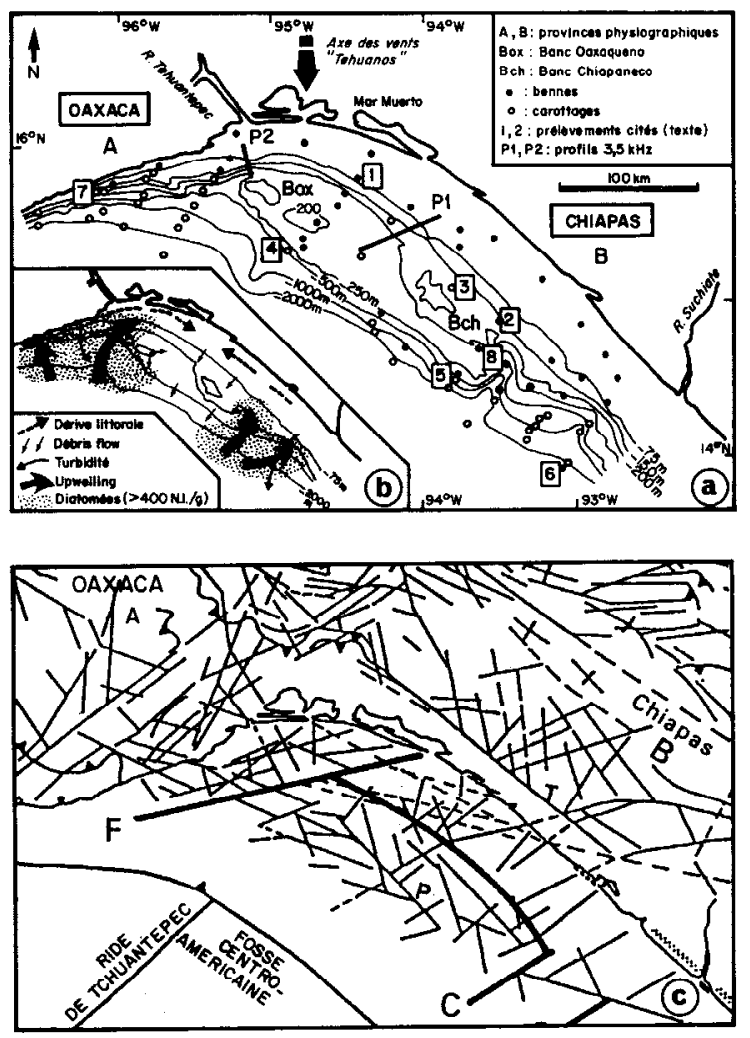

Figure 2

a) Bathymétrie du golfe de Tehuantepec (INEGI) : localisation des profils sismiques $3.5 \mathrm{kHz}$ et des prélèvements (bennes et carottages) au cours de la mission PROGEMA I. - 1: BO20, 2: KS 151, 3: KS 148, 4: KS 126, 5 : KS 154, 6: BO71, 7 : KS 104, 8: KS 160. b) Répartition, en nombre d'individus par gramme, des diatomées (Thalassionema nitschioides var. parva et Nitzschia kerguelensis): upwellings préferentiels dans les canyons. c) Traits structuraux du Mexique méridional (Boumaggard, 1994): en domaine continental, structures compilées à partir de nombreux travaux; en domaine marin structures élaborées à partir de l'analyse morpho-structurale (reliefs, réseau d'entailles, anomalies de drainages, etc). F : décrochement sénestre $\mathrm{N} 70^{\circ}$ séparant les provinces physiographiques Nord occidentale et Sud orientalc; $\mathrm{C}$ ct $\mathrm{F}$ : accidents cassants à l'origine de canyons.

a) Bathymetric framework of the Gulf of Tehuantepec: location of $3.5 \mathrm{kHz}$ seismic profiles, surface samples and core stations during the PROGEMA I cruise (1989). I: BO20, 2: KS 151, 3: KS 148, 4: KS 126, 5: KS 154, 6: BO71, 7: KS 104, 8: KS 160. b) Distribution of diatoms (Thalassionema nitschioides var. parva and Nitzschia kerguelensis) abundance in the sediments, expressed in terms of number per gram of the sediment. Note preferential upwelling in canyons. c) Structural features of Southern Mexico (Boumaggard, 1994): compiled structures in domain continental; structures elaborated from morphostructural analysis in the marine field. F: wrench fault $N 70^{\circ}$ isolating the northwestern and southeastern physiographic provinces, $C, F$ : breaking accidents related to canyons.

nante de coulissements sénestres assurant le découplage entre plaque Nord-Américaine et plaque Caraïbe (Fig. $2 c$ ).

\section{Morphostructure de la plate-forme}

L'analyse morphostructurale de la plate-forme (Boumaggard et al., 1993; Bobier et al., 1993; Boumaggard, 
1994) révèle la présence de deux grandes provinces physiographiques distinctes, l'une nord-occidentale (A Fig. 2a), l'autre sud-orientale (B Fig. 2a) séparées par un décrochement sénestre majeur $\mathrm{N} 70^{\circ}$ (F Fig. $2 c$ ). Le tracé de la côte sud-orientale de l'Etat de Oaxaca est da à un accident de même direction alors que le littoral occidental est parallèle à une faille $\mathrm{N} 120^{\circ}$ (Carfantan, 1986).

La province nord-occidentale, correspond au plateau continental du bloc de Oaxaca. La plate-forme d'abrasion sous-marine très étroite (pente de 2 a $5 \%$ ), la pente continentale abrupte $(6 \%)$ et entaillee de nombreux canyons guidés par des accidents tectoniques $\mathrm{N} 145^{\circ}$ (Boumaggard et al., 1993) montrent que l'on a ici une marge en cours de surrection.

La province sud-orientale, correspond au plateau continental de la Sierra de Chiapas. Plusieurs sous-unités physiographiques y sont reconnues (Fig. 2a):

- une plate-forme interne, jusqu'à $-75 \mathrm{~m}$, à morphologie très uniforme;

- une rupture de pente entre -75 et $-100 \mathrm{~m}$;

- une plate-forme externe qui s'étend de $-100 \mathrm{~m}$ jusqu'à $-250 \mathrm{~m}$ à morphologie beaucoup plus contrastée du fait de la présence de hauts-fonds gréseux séparés par des dépressions d'origine tectonique (Boumaggard et al., 1993; Boumaggard, 1994) et de ruptures de pente situées à $-120 / 130 \mathrm{~m}$ et $-225 / 250 \mathrm{~m}$, parallèles à la côte actuelle.

Les ruptures de pente de $-75 /-100 \mathrm{~m}$ et de $-225 /-250$ $\mathrm{m}$ correspondent à des discontinuités bien marquées dans la morphologie de surface (Boumaggard et al., 1993) et très probablement enracinées en profondeur (Boumaggard, 1994). La première, bien que scellée par la progradation des sédiments quaternaires, correspondrait à une faille active jalonnée par plusieurs êpicentres de séismes récents (Dengo, 1983). Celle de $-225 /-250 \mathrm{~m}$ marque la limite occidentale d'une troncature d'érosion d'âge miocène (Sanchez-Barreda, 1981).

Au delà, la marge présente un talus, entaillé par des canyons $\mathrm{N} 45^{\circ}$ à $\mathrm{N} 70^{\circ}$ parfois profonds $(-1700 \mathrm{~m})$ et à pente très forte $(6$ à $10 \%)$, qui plongent jusqu'à la fosse mésoaméricaine. L'implantation des canyons est déterminée par le réseau de failles qui découpe la plate-forme et la marge (Bobier et al., 1993) et s'inscrit dans le même système que celui qui découpe l'isthme de Tehuantepec (Fig. 2c).

\section{Forçage de l'hydrologie et transferts de matières}

\section{Le forçage des courants de surface (Fig. 1)}

En hiver les courants de surface subissent un forçage intermittant (Barton et al., 1993) par un violent vent de terre, de secteur Nord, les "Tehuanos " Ce courant atmosphérique est engendré (Hurd, 1929; Roden, 1961) par l'apparition, dans le golfe du Mexique, d'un champ de hautes pressions, alors que les pressions demeurent basses dans la zone équatoriale du Pacifique. Ces vents accélérés par effet «Venturi » (Clarke, 1988) atteignent une vitesse de $25 \mathrm{~m} / \mathrm{s}$ (Alvarez et al., 1989) Iorsqu'ils traversent l'isthme de Tehuantepec en empruntant des dépressions tectoniques N-S. Dans le golfe de Tehuantepec, ils induisent perpendiculairement à la côte, une dépression de $200 \mathrm{~km}$ de large et de $500 \mathrm{~km}$ de long ou circule un courant N-S (Roden, 1961 ; Lavin et al., 1992) froid, violent et intermittent correspondant à de véritables jets d'eau (type « squirt » de Ramp et al., 1991), et même de type « supersquirt" (Barton et al., 1993). Ce courant entraîne, accélère et dévie vers le Sud les courants de dérive littorale opposés qui convergent dans le golfe (Alvarez et al., 1989; Lavin et al., 1992).

Venant du Sud-Est, le courant de Costa-Rica qui circule parallèlement aux structures du Massif de Chiapas atteint $100 \mathrm{~cm} / \mathrm{s}$ et forme une gyre anticyclonique alors qu'à l'Ouest du golfe se forme une gyre cyclonique à partir du courant $(100 \mathrm{~cm} / \mathrm{s})$ de dérive nord-occidentale orienté, SW-NE, parallèlement au littoral, déterminé par la présence d'une faille $\mathrm{N} 70^{\circ}$ (Carfantan, 1986).

En été les vents sont faibles ( 5 à $10 \mathrm{~m} / \mathrm{s})$. Le courant de Costa-Rica balaie l'ensemble du golfe (Roden, 1961).

Conséquences sur la dérive littorale et les transferts de matières associés (Fig. 2a).

La période hivernale de vents forts coïncide avec la saison humide durant laquelle les apports sédimentaires par les fleuves sont maximaux (Carranza-Edwards, 1989). Ces apports fluviatiles, repris par les deux gyres, favorisent la formation de fleches littorales qui convergent au niveau de la Mer Morte.

\section{Le forçage tectonique de la circulation en profondeur}

Cette circulation est aussi largement influencée par les effets du forçage hivennal des circulations de surface liées à la structure tectonique de l'isthme (Fig. 2a). En effet le départ des masses d'eaux entrainées vers le Sud par les «Tehuanos » est compensé par l'apport d'upwellings (Roden, 1961 ; Stumpf, 1975 ; Carranza-Edwards et al., 1989; McCreary et al., 1989; Morales- Garza, 1990; Perez-Cruz et Machain, 1990; Lavin et al., 1992; Barton et al., 1993; Molina-Cruz et Martinez-Lopez, 1994). Ces remontées d'eaux profondes sont accompagnées en surface par:

- des zones à températures très basses $\left(10^{\circ} \mathrm{C}\right.$ selon McCreary et al., $1989 ; 8^{\circ} \mathrm{C}$ selon Barton et al., 1993) et la mise en place d'eaux à température inférieure à $14^{\circ} \mathrm{C}$ dans la partie sud-orientale du golfe (Lavin et al., 1992).

- de faibles teneurs en oxygène $(5,03 \mathrm{ml} / \mathrm{l}$ selon Perez-Cruz et Machain, 1990) et une concentration minimale inférieure à $0,5 \mathrm{ml} / 1$, entre 100 et $1300 \mathrm{~m}$ (Carranza-Edwards et al., 1989)

Conséquences sur la distribution des fux de mutière associés aux upwellings

L'influence de ces courants se traduit par:

- des noyaux de sables à fortes concentrations en phosphates (Carranza-Edwards et al., 1989; Morales- 


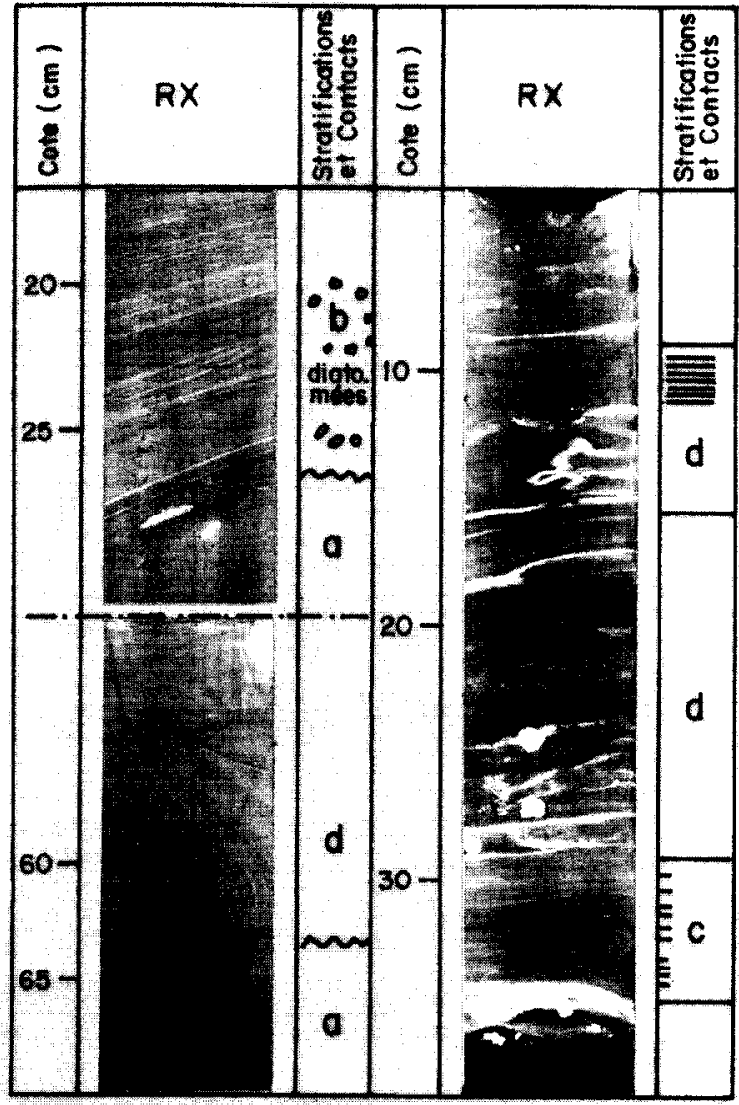

LEGENDE.

a - Vases hémipélagiques

b - Laminites a diatomées (Thalassionema nitschioides var. parva)

c - Vases laminées silfeuses a argileuses

d. Dépôts silteux (glissement)

m Surface d'erosion

- Contact graduel

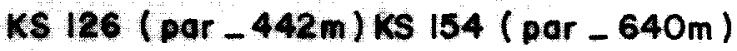

Figure 3

Mise en évidence des milieux de dépôts attribuables à des upwellings dans la province sud-orientale.

Demonstration of deposit environments associated with upwellings in the southeastern province.

Garza, 1990) localisés en particulier (Fig. 2c) sur les hauts-fonds d'origine tectonique (Boumaggard, 1994);

- la présence, dans les prélèvements que nous avons étudiés, de deux assemblages de radiolaires différents de part et d'autre du courant superficiel engendré par les «Tehuanos » (Molina-Cruz et Martinez-Lopez, 1994):

au Nord-Ouest, un assemblage introduit en hiver dans le golfe par le courant de Californie (Molina-Cruz, 1988 ; Molina-Cruz et Martinez-Lopez, 1994) puis repris par le courant de dérive qui longe le littoral du bloc de Oaxaca avant d'être dévié vers le Sud par les «Tehuanos».

au Sud-Est un assemblage de formes caractéristiques d'eaux tropicales et subtropicales (Molina-Cruz, 1988) introduites dans le Golfe de Tehuantepec principalement en été, par le courant de Costa-Rica. La présence (MolinaCruz, 1984) de Acrosphaera murrayana, caractéristique des upwellings du Pérou mais peu représentéc dans les caux tropicales et subtropicales, prouve l'existence d'un courant d'upwelling, important en hiver, dans cette partie du golfe (Molina-Cruz et Martinez-Lopez, 1994).

Par ailleurs, nos carottes présentent de fréquentes laminites à diatomées telles Thalassionema nitschioides var. parva caractéristiques des zones d'upwelling (Abrantes, 1991) et Nitzschia kerguelensis provenant de l'océan Austral (Pichon et al., 1992). La présence (Fig. 3), dans les sédiments de surface de ces diatomées signalées jusqu'au large de Los Angeles (Sautter et Sancetta, 1992), témoigne d'upwellings alimentés à partir de la circulation générale du fond du Pacifique. Ces upwellings remontent jusqu'à la plate-forme interne, canalisés par les canyons. Cette focalisation du drainage est responsable de l'individualisation d'aires à foraminifêres (Bolivinidae) et d'aires à diatomées (Fig. $2 b$ ).

LES GRANDS DOMAINES DE SÉDIMENTATION, REFLETS DU CONTRÔLE TECTONIQUE DES PROCESSUS ET DES FACIÈS SÉDIMENTAIRES

Complétant celles réalisées antérieurement par l'ICMyL (Carranza-Edwards, 1980, 1986 et 1989; CarranzaEdwards et al., 1989; Morales-Garza, 1990) nos ćtudes permettent de présenter une synthèse (Fig. 4) des principaux faciès de la couverture sédimentaire entre 0 et $-200 \mathrm{~m}$. Au delà, jusqu'à $-2250 \mathrm{~m}$, les données sont 


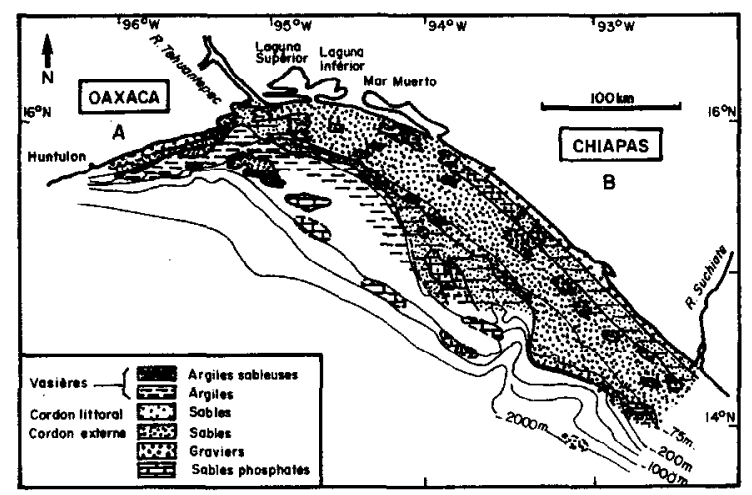

Figure 4

Répartition des faciès lithologiques de surface (Morales-Garza, 1990; modifié).

Surface lithological facies distribution (Morales-Garza, 1990; modified).

ponctuelles, toutefois elles permettent de reconnaître des sédiments relativement homogènes tant par leurs faciès que par leurs modes de mise en place. La répartition des faciès reconnus met en évidence l'étroit parallélisme entre les provinces sédimentaires et les domaines morphostructuraux du golfe (Boumaggard et al., 1993). Aussi les caractères de la sédimentation seront présentés selon les domaines physiographiques.

\section{Province sud-orientale}

\section{Plate-forme interne $(0 \grave{a}-75 \mathrm{~m})$}

Les analyses sédimentologiques (Fig. 5) et morphostructurales ainsi que la coupe sismique $3,5 \mathrm{kHz}$ (Fig. 6) réalisée à la limite des plates-formes interne et externe, montrent le développement d'un cordon sableux interne ou littoral et d'un cordon externe encadrant une vasière (Fig. 4) située entre 10 et $30 \mathrm{~m}$ de profondeur.

La vasière correspond aux dépôts de proche plate-forme, de type " near shore mud-belt ", recensés par Mac Cave (1972) qui a noté que ces formations sont très souvent localisées entre une zone interne de sables récents et une zone externe de sables généralement fossiles. Ici, cette vasière est liée aux apports à smectites $(50$ à $70 \%)$ + illitekaolinite, par le fleuve Suchiate.

Le cordon sableux littoral, reconnu jusqu'à $-10 \mathrm{~m}$, prolonge sur la plate-forme interne les cordons sableux (10 $\mathrm{m}$ d'épaisseur) décrits autour des lagunes supérieure et inférieure (Cromwell, 1975). Il est formé de sables transportés par la dérive littorale SE-NW (Lavin et $a l$, 1992) depuis le fleuve Suchiate à l'Est jusqu'au décrochement $\mathrm{N} 70^{\circ}$ (Fig. 3).

Le cordon sableux externe (Fig. 4, Fig. 6) développé de $-30 \mathrm{~m}$ à $-70 \mathrm{~m}$, présente un modelé uniforme; il a fait l'objet de prélèvements par benne. La faiblesse de la pénétration des ondes (forte impédance acoustique de l'interface eau-sédiment et forte dispersion des ondes)
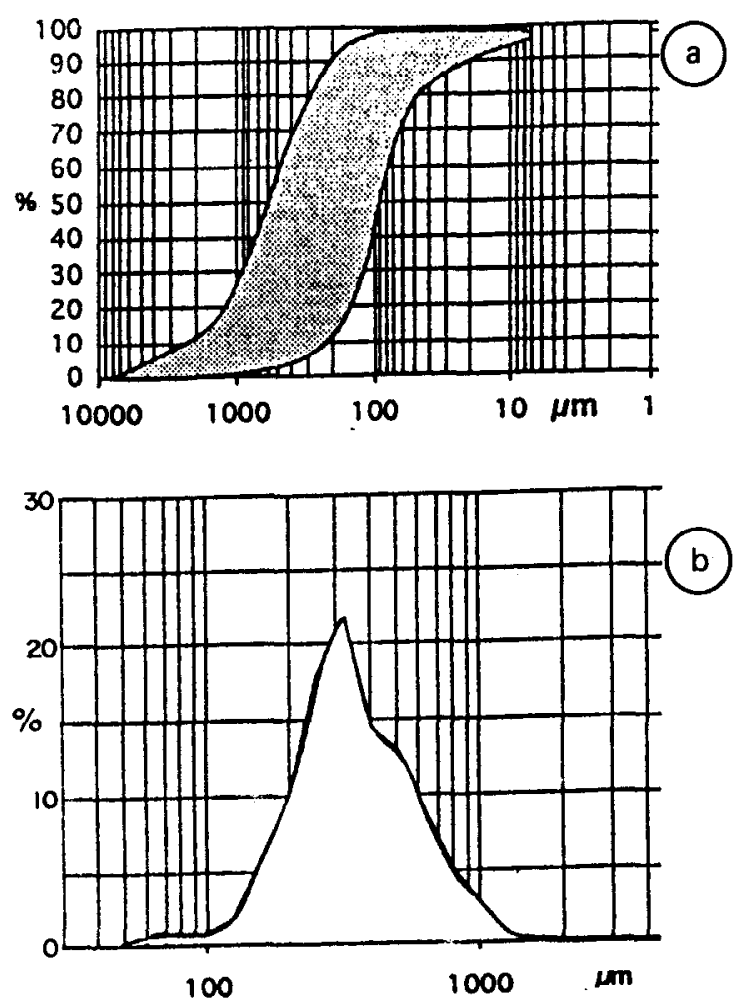

Figure 5

Courbes granulométriques représentatives des sables de la plateforme interne. a) Courbes cumulatives enveloppes. b) Courbes de fréquence.

Representative curves of the granulometry of the inner platform sands. a) Cumulative curves. b) Frequency curves.

n'a pas permis de mettre en évidence la présence d'éventuelles structures sédimentaires ni d'évaluer avec précision l'épaisseur de ce cordon. L'observation du profil (Fig. $6 b$ ) permet d'estimer que son épaisseur peut dépasser localement $30 \mathrm{~m}$. L'analyse granulométrique montre que ces cordons sont constitués de sables coquilliers et/ou de silts grossiers, très peu classés (déviation standard $=2,3$ ) sauf pour les particules les plus grossières (Skewnes $=1,81$ ). Les courbes cumulatives sont de type parabolique et les courbes de fréquence sont unimodales avec prédominance des particules proches de $300 \mu \mathrm{m}$ et léger enrichissement en particules de $500 \mu \mathrm{m}$. (Fig. 5). Ce cordon présente deux particularités:

- une série de noyaux de sables et graviers le long de l'isobathe $-40 / 45 \mathrm{~m}$ (Fig. 4);

- une rupture de pente marquée $(3 \%)$ à sa limite externe, de -70 à $-75 \mathrm{~m}$.

L'examen au microscope électronique à balayage (MEB), complété par un spectre d'analyse spectrale $X$, de grains de sable prélevés à -45 et $-75 \mathrm{~m}$, permet d'identifier deux stocks: d'une part, des cendres volcaniques à dépôts de silice emprisonnant des diatomées; d'autre part, des quartz à dépôts siliceux piégeant des coccolithes. Les deux stocks indiquent que ces sables correspondent à des environnements de tendance estuarienne à influences 


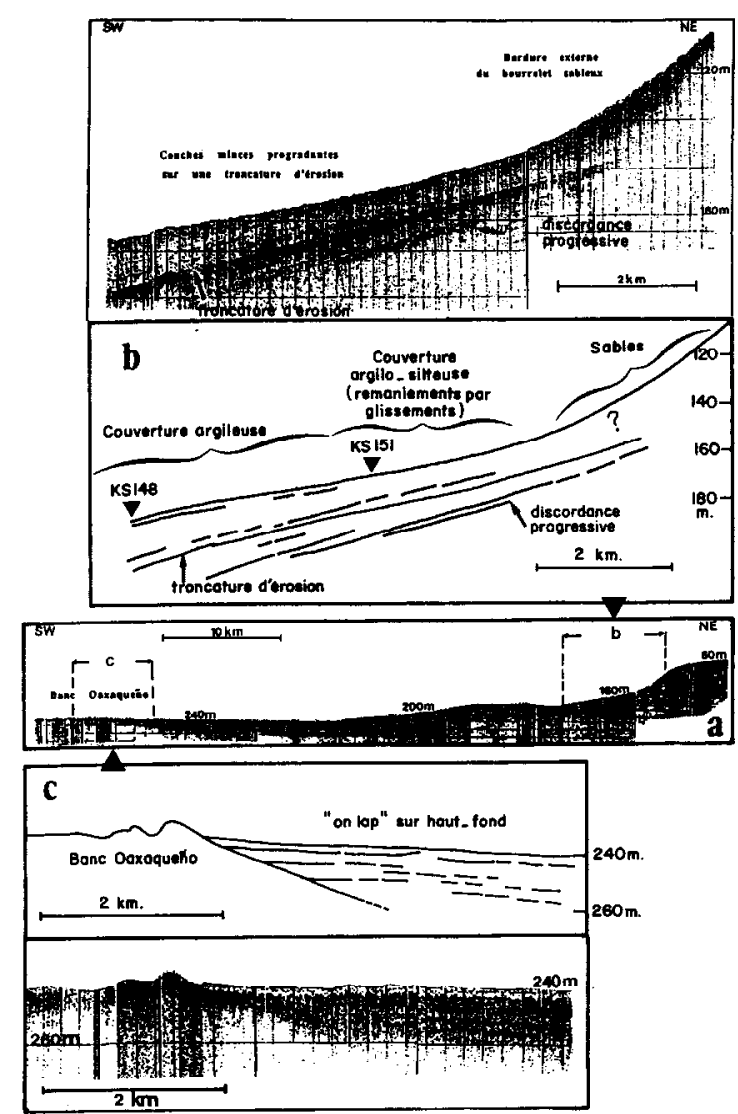

Figure 6

Progradation des dépôts récents dans la province sud- orientale. a) profil sismique $3.5 \mathrm{kHz}$ général (P1 : localisation sur la Fig. 2); b et c) interprétations des sections $\mathrm{b}$ et $\mathrm{c}$ : noter le biseau d'aggradation sur le haut-fond et la troncature d'érosion sur la bordure externe de la plate-forme interne.

Progradation of recent deposits in the southeastern province. a) general $3.5 \mathrm{kHz}$ profile ( $P$ I profile location in Fig. 2 ). $\mathbf{b}$ and $\mathbf{c}$ ) section interpretation: note the "onlap" on the shoals and the "offlap" on the external edge of the inner continental shelf.

marines très peu marquées (Le Ribault, 1975). Ces dépôts relictes, associés à des lambeaux de graviers, confirment le stationnement de la mer et l'installation de paléo-rivage à -45 et $-75 \mathrm{~m}$. Ils peuvent être interprétés comme la signature d'une transgression rapide sur un substrat sableux.

Pour les deux cordons, le cortège des minéraux lourds est dominé par les pyroxènes (hypersthène $30-74 \%$, augite 18 $41 \%$ ) et les amphiboles (hornblende basaltique 13-30\%). Les minéraux présents sont frais et bien conservés, ils sont drainés par le fleuve Suchiate depuis le Massif andésitique de Chiapas (Carranza-Edwards, 1989). La distribution sur la plate-forme interne des sédiments terrigènes est donc assurée par la dérive littorale associée au courant de CostaRica. Elle traduit la mise en place de termes transgressifs et de haut niveau marin asociés à la remontée eustatique postglaciaire.
La limite entre les plates-formes interne et externe $(-75 /-100 \mathrm{~m})$ est caractérisée par un placage silto-sableux localement recouvert par une séquence argileuse fine qui drape aussi la dépression qui sépare le cordon externe des hauts-fonds phosphatisés. L'examen de la section sismique $3,5 \mathrm{kHz}$ (Fig. $6 b$ ) réalisée sur cette rupture de pente semble montrer que, sous cette couverture argileuse, le cordon présente les caractéristiques suivantes:

- il recouvre une surface d'érosion qui se prolonge sous la plate-forme externe et recoupe une série marquée par des discordances progressives;

- le bourrelet sableux est relayé vers le large, sans discontinuité visible, par une série argilo-silteuse accumulée en couches minces progradantes sur la troncature d'érosion; cette zone de transition avec passage latéral de faciès semble toutefois drapée par la couche argileuse superficielle.

I'importance de la pente et l'activité séismique (Heubeck et Mann, 1991) provoquent de fréquentes déformations des sédiments, combinant glissement et thixotropie comme nous avons pu les observer sur les carottes et sur les clichés R.X. provenant de cette zone (carotte KS 151, Boumaggard, 1994).

Le cordon externe peut donc être considéré : soit comme un front deltaïque antérieur au placage argileux superficiel ; soit comme un niveau de stationnement marin lors de la remontée postglaciaire. La continuité et la régularité de ce bourrelet sableux parallèlement à la côte nous conduit à privilégier la seconde interprétation. De telles phases de stabilisation du niveau marin ont déjà été reconnues sur les marges stables (Curray, 1964 pour la marge atlantique nordaméricaine; Pirazzoli, 1991 pour la marge européenne) et sur des marges ayant subi une subsidence de quelques mètres sous l'action d'une néotectonique distensive comme les marges atlantique du Maroc (Cirac et al., 1993) et pacifique de Colombie (Correa, 1996). Ces paléorivages semblent liés au maximum de la régression würmienne $(-120 / 130 \mathrm{~m})$ et à des phases de stabilisation $(-75$ et $-45 \mathrm{~m}$ ) lors des périodes de refroidissement du climat (Duplessy et al., 1970).

En résumé, la plate-forme interne est constituée par l'accumulation, sur une surface d'érosion, d'un cordon interne actuel et d'un cordon externe fossile, témoins de la remontée eustatique postglaciaire et de la faiblesse des mouvements verticaux à l'échelle de temps considérée. Ils constituent deux termes du système intervalle transgressif récent-prisme de haut niveau marin actuel. On peut considérer que le prisme d'accrétion tectonique, qui borde la plate-forme sud orientale, a atteint son profil d'équilibre et que la marge du golfe de Tehuantepec se comporte comme une marge passive.

\section{Plate-forme externe et talus continental}

La morphologie de la plate-forme externe est contrastée. Des hauts-fonds (Bancs Oaxaqueño, - $175 \mathrm{~m}$ et Chiapaneco $-170 \mathrm{~m}$, Fig. $2 a$ ) sont séparés de la plate-forme interne par des dépressions internes $(-200 \mathrm{~m})$. Une rupture de pente autour de $-250 \mathrm{~m}$ sépare cette plate-forme du talus 
continental qui plonge avec une pente moyenne de $10 \%$, directement jusqu’à la fosse. Ce talus est incisé dans le secteur oriental, par deux canyons $\mathrm{N} 45^{\circ}$ d'origine tectonique.

Les hauts-fonds sont constitués de grès phosphatisés et couverts par les produits de démantèlement (graviers de grès et sables phosphatisés au Miocène ou en voie de phosphatisation) associés à des bioclastes grossiers. Ces démantèlements n'ont pu se produire que dans des conditions hydrodynamiques plus actives qu'actuellement. Ils témoignent d'une période de bas niveau marin miocène à pléistocène. La sédimentation actuelle est nulle ou réduite à des placages argileux piégés dans de petites cuvettes de quelques mètres de profondeur.

Les dépressions internes sont alimentées dans leur zone axiale profonde par des apports silto-argileux hémipélagiques (carotte Ks 148; Boumaggard, 1994) interrompus sur les flancs (Carotte KS 151 ; Boumaggard 1994) par des apports par glissements. Ces dépôts présentent à la base un biseau d'aggradation sur les hauts-fonds rocheux (Fig. 6c). Les couches inférieures du remplissage et les sables qui les relaient dans le cordon externe, correspondent à un intervalle trangressif postglaciaire. La faiblesse du drapage superficiel actuel et l'augmentation de la teneur en silts vers le sud-est (Fig. 4) sont sans doute dus au fait qu'unc partie des sédiments fins de surface est reprise par les courants d'upwelling (Fig. 2b) puis transportée vers le large.

A la rupture de pente $(-225 /-250 \mathrm{~m})$ apparaissent des graviers $(2 \mathrm{~cm}$ ) et des sables grossiers (Fig. 4) formés essentiellement de nodules de calcaires oolithiques et pisolithiques phosphatés miocènes (Boumaggard, 1994). La bathymétrie de ces dépôts permet de les rattacher au prisme de bas niveau marin miocène reconnu en sismique (Sanchez-Barreda, 1981). Ils peuvent donc être interprétés comme des sédiments reliques.

La partie supérieure du talus, montre une couverture sédimentaire constituée de vases silto-sableuses (moyenne 36-83 $\mu \mathrm{m})$ carbonatées $\left(\mathrm{CaCO}_{3}=31\right.$ à $57 \%$ ) à foraminifères.

La sédimentation dans cette partie qui présente une forte pente (6 à $10 \%$ ) a été étudiée par deux carottes (Fig. 3) prélevées par $-422 \mathrm{~m}$ au large du Banc Oaxaqueño (carotte Ks 126 ; longueur $92 \mathrm{~cm}$; $\mathrm{n}^{\circ} 4$ Fig. $\left.2 a\right)$, et par $(-610 \mathrm{~m}$ au large du Banc Chiapaneco (carotte 154 ; longueur $150 \mathrm{~cm}$; $\mathrm{n}^{\circ} 5$ Fig. $2 a$ ). Les sédiments sont constitués par:

- des vases hémipélagiques (a, Fig. 3);

- des vases laminées (laminites à Thalassionema nitschioides var. parva) lićes aux courants d'upwclling (b, Fig. 3);

- des vases laminées silteuses à argileuses (c Fig. 3) qui peuvent être interprétées soit comme des turbidites de particules fines (termes T1 à T7 et T8 bioturbé; Stow et Shanmugan; 1980); soit comme des intervalles supérieurs (termes $d$ et $e$ ) de la séquence de Bouma (1962), bien qu'il soit difficile d'établir une filiation directe entre les vases non carbonatées du talus et les vases carbonatées sus-jacentes; soit comme des sédiments associés à des courants de contours; l'absence d'un maillage de profils bathymétriques et le nombre restreint de prêlèvements ne permettent pas de mettre en évidence de gradients granulométriques caractéristiques de ce type de corps sédimentaire;

- des dépôts de silts ( $d$, Fig. 4 ) provenant du glissement, sur de faibles distances, de couches fortement thixotropiques.

Les canyons. L'analyse des coupes sismiques (SanchezBarreda, 1981; Boumaggard, 1994) a révélé l'existence d'une phase de creusement de canyons au Miocène moyen puis de colmatage du Miocène supérieur au PlioQuaternaire. Les axes de drainage étant guidés par des cisaillements, compte tenu de l'orientation des canyons actuels par rapport aux fleuves, on peut penser que ceuxci ont réemprunté le cours des paléocanyons lors du plus récent abaissement eustatique mais que le prisme de haut niveau marin a comblé et masqué cette connexion.

Les canyons sont alimentés par:

- des dépôts hémipélagiques formés d’argiles bioturbées à foraminifères pélagiques;

- des écoulements turbiditiques composés de matériaux fins disposés en lamines montrant des surfaces d'érosion, de réactivation et des niveaux riches en microfaune benthique issue de la plate-forme interne ; un seul prélèvement (BO71, par $-2050 \mathrm{~m}$ dans l'axe du canyon), constitué de sables granulométriquement et minéralogiquement identiques à ceux de la plate-forme interne, indique la présence de courants de turbidité à capacité de transport élevée, Les données disponibles ne permettent pas de dire si cette dynamique est exceptionnelle ou si un cône sous-marin profond se développe.

- des vases, riches en matière organique, à laminites de diatomées (Thalassionema nitschioides var. parva) et faunes pyritisées traduisant l'action des courants d'upwelling.

Sur la zone interfluve séparant les canyons, les dépôts, constitués par des vases argilo-silteuses terrigènes (argiles $>65 \%$ ) ne montrent pas de surface érosive. Les faciès et les microgranulométries reflètent une sédimentation par décantation d'un nuage néphéloïde. La microfaune est dominée par des formes bien conservées de foraminifères planctoniques $(80 \%)$ associés à des foraminifères benthiques profonds et à des formes souvent brisées issues d'horizons supérieurs. On observe fréquemment des vases laminées associées à un milieu réducteur (foraminifères pyritisés, forte odeur de $\mathrm{SH}_{2}$ ) riche en matière organique fraîche (abondance des Bulimina exilis qui témoignent de l'influence des courants d'upwelling; Caralp, 1989).

Sur la pente il y a donc une accumulation de matériaux issus du plancton, complétée par des apports détritiques qui suivent la pente en direction de la zone profonde, et développement d'une microfaune benthique alimentée en matières nutritives par les courants d'upwelling.

\section{Province nord-occidentale}

Dans cette province d'extension réduite, l'échantillonnage est limité. Toutefois, le contexte morpho-tectonique différent influe sur la sédimentation quaternaire. 


\section{La plate-forme}

A l'Est, au droit du fleuve Tehuantepec, la plate-forme assez large est réduite à la plate-forme interne où l'on trouve des sables bioclastiques moyens (minéraux lourds cardinaux: grenats, de 40 à $50 \%$ et amphiboles de 18 à $50 \%$ ). Ce cortège, identique à celui décrit par CarranzaEdwards (1980) à l'embouchure du fleuve Tehuantepec et sur les flèches littorales de cette province, est issu de la Sierra de Oaxaca. L'apparition de chlorite (10 à 20\%) dans une association smectites-illite-kaolinite différencie également le cortège des minéraux argileux de celui du domaine Sud-Oriental.

La coupe sismique $3,5 \mathrm{kHz}$ (Fig. 7) révèle, vers $-130 \mathrm{~m}$, l'accumulation, sur une troncature d'érosion, de réflecteurs de forte amplitude recouverts par les dépôts fins actuels. Ces constructions sédimentaires qui traduisent l'alternance de phases d'accumulation et de creusement, peuvent être rattachées à un prisme littoral immergé associé à la régression glaciaire würmienne reconnue dans la province sud orientale.

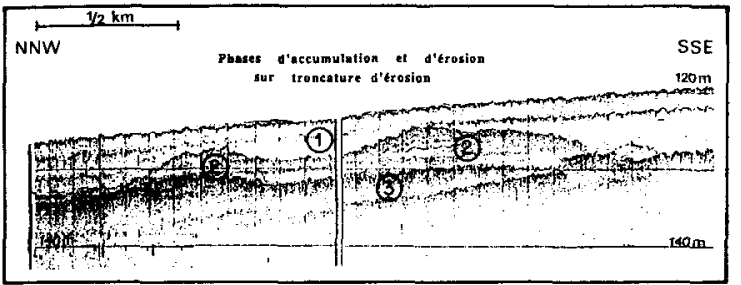

Figure 7

Phases d'accumulations sédimentaires dans la province nord occidentale (P2 localisation sur la Figure 2), 1) dépôts argileux récents, 2) cordons sableux fossiles, 3 ) troncature d'érosion vers $-130 \mathrm{~m}$.

Sedimentary accumulation phases in the northwestern province ( $P 2$ profile location in Fig. 2), 1) recent clayey deposits, 2) fossil sundy bars, 3) erosional truncation near $130 \mathrm{~m}$ water depth.

- une série de noyaux de sables et graviers le long de l'isobathe $-40 / 45 \mathrm{~m}$ (Fig. 4);

A l'Ouest, les profils bathymétriques abrupts prolongent ceux de l'arrière-pays.

$\mathrm{La}$ plate-forme est réduite à une étroite plate-forme d'abrasion. Les dépôts, du fait de la réduction extrême de l'espace disponible pour la sédimentation sont réduits à des placages de sables jusqu'à $-75 \mathrm{~m}$, puis des sables argileux et des argiles sableuses jusqu'à $-200 \mathrm{~m}$. Les constituants minéralogiques sont identiques à ceux de la zune Est, seul se note un enrichissement en grenats $(70 \%)$.

\section{Le talus}

La sédimentation est argilo-silteuse, riche en débris ligneux grossiers et en micas avec des horizons grano-classés de sables, fins à moyens, ou de silts grossiers qui pourraient correspondre à des glissements turbitiques (KS 104, Fig. 8). La nature des cortèges minéralogiques montrent que ces matériaux sont apportés par le fleuve Tehuantepec lors des périodes de crues (débit atteignant $1368725 \mathrm{~m}^{3} / \mathrm{an}$; Carranza-Fdwards, 1980) car en période d'étiage ce fleuve est réduit à l'état de ruisseau.

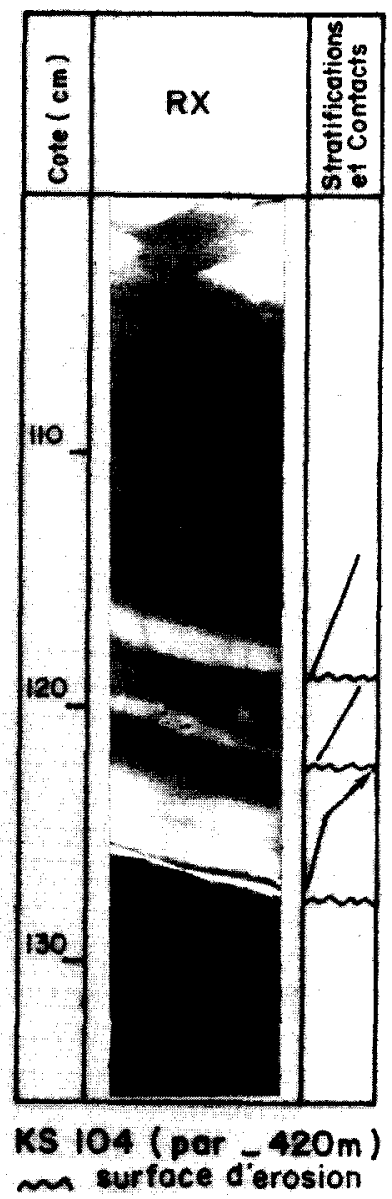

Figure 8

Mise en évidence d'arrivées sableuses turbiditiques au sommet du talus de la province nord-occidentale.

Demonstration of turbiditic sand deposits on the continental slope of the northwestern province.

Dans l'axe d'un canyon, (vers $-1210 \mathrm{~m}$ ) la présence de sables identiques à ceux de la plate-forme témoignent d'une alimentation par courants gravitaires.

De même, la présence par $-2100 \mathrm{~m}$ de niveaux sablosilteux et de débris récifaux provenant de Huntulon, où se trouvc le seul récif connu dans cette zone, prouvent l'exjstence d'écoulements gravitaires.

Dans ce secteur, la morphologie, les reliefs très accusés de l'arrière-pays et le régime pluviométrique (CarranzaEdwards, 1980) ne permettent pas l'installation d'un réseau hydrographique de type chenalisant important, mais favorisent la création d'un réseau en tresses et de nombreux ruisseaux. Cette morphologie très abrupte se retrouve en domaine marin et les données disponibles ne permettent pas de savoir si les courants gravitaires sont chenalisés par les canyons ou si la forte pente induit une alimentation 
gravitaire générale en nappe. Ce type d'alimentation directe des zones profondes a déjà été noté dans le Détroit de Makassar à partir de la marge active de Sulawesi (Gayet et al., 1990) et dans la partie Nord de la marge active Pacifique colombienne (Correa, 1996).

\section{CONCLUSION}

Sur la plate-forme du golfe de Tehuantepec, la distribution des dépôts récents résulte d'une interaction entre l'eustatisme et un double contrôle tectonique. La présence de dépôts associés à des périodes de bas et de haut niveaux marins montre que sur ce type de marge, malgré l'activité sismique, les phénomènes liés à l'eustatisme peuvent être enregistrés. Toutefois la distribution des cortèges sédimentaires est contrôlée par la tectonique.

Contrôle par la tectonique de l'isthme de l'hydrologie et des provinces minéralogiques

La présence d'un réseau de failles conjuguées $\mathrm{N} 120^{\circ}$ et $\mathrm{N} 45^{\circ}$ découpant l'isthme et la plate-forme de Tehuantepec détermine la forme du littoral et induit le développement de deux courants de dérives convergents.

L'existence de couloirs tectoniques Nord-Sud recoupant l'isthme provoque une accélération de la circulation atmosphérique entre les golfes du Mexique et de Tehuantepec, le forçage des vents «Tehuanos » qui en résulte induit à son tour le forçage des circulations.

En surface, ces vents transforment les courants de dérive en deux gyres qui assurent la dispersion des produits détritiques apportés depuis la Sierra de Oaxaca (fleuve Tehuantepec) et depuis le Massif de Chiapas (fleuve Suchiate). Les caractéristiques minéralogiques différentes des sédiments dispersés accentuent l'individualisation de part et d'autre d'un décrochement $\mathrm{N} 70^{\circ}$ de deux provinces physiographiques héritées d'une évolution géodynamique complexe (Stephan ot al., 1990).
Les circulations de fond sont également affectées par ces vents qui favorisent l'apparition de processus d'upwelling dans les canyons et sur la plate-forme.

Contrôle de la morphologie par l'interaction eustatismetectonique de la plate-forme

La ligne de côte de la province nord-occidentale (marge du bloc de Oaxaca) correspond à des falaises plongeant directement dans le Pacifique ou se raccordant à une plateforme réduite de type plate-forme d'abrasion. Au large du fleuve Tehuantepec où la plate-forme est plus large, s'édifie un prisme de progradation d'étendue limitée et il semble bien qu'un prisme de bas niveau ait été façonné au Würm.

Sur le littoral de la province sud-orientale (plateau continental du massif de Chiapas), la plate-forme interne correspond à un prisme de haut niveau marin, limité vers le large par une rupture de pente. La plate-forme externe est façonnée par la tectonique, avec des dépressions formant pièges à sédiments et des hauts-fonds, balayés par des upwellings, où se concentrent les produits de la phosphatogenèse.

Des canyons guidés par des failles actives drainent de faibles écoulements turbiditiques, mais également les courants d'upwelling en raccordant la plate-forme au système de la circulation générale profonde. Cette circulation détermine la différenciation en zones riches en foraminifères d'eaux froides et zones à oxygène minimum enrichies en tests siliceux.

Enfin, la plate-forme du golfe de Tehuantepec a enregistré trois niveaux de stationnement marin $(-45 \mathrm{~m},-75 \mathrm{~m}$ et $-130 \mathrm{~m}$ ). L'absence de datation ne permet pas de préciser l'âge de ces niveaux qui toutefois correspondent aux données de la remontée eustatique postglaciaire et de la période de bas niveau marin würmienne classiquement reconnues sur les marges passives. Cette marge active semble avoir atteint son profil d'équilibre et présenter des caractères de type marge passive en bordure de la plaque Caraine.

\section{REFERENCES}

\begin{abstract}
Abrantes F.F. (1991). Variability of upwelling of NW Africa during the latest Quaternary: diatoms cvidence. Paleoceanology 6, 431-460.

Aguayo-Camargo J.E., C. Marin (1987). Origen y evolucion de los rasgos morfotectonicos poscretacicos de México. Bol. Soc. Geol. Mexicana, Tomo XLVIII, 2, 17-39.
\end{abstract}

Alvarez L.G., A. Badan-Dangon, A. Valle (1989). On coastal currents off Tehuantepec. Est. Coast. Shelf Sci. 29, 1, 89-96.

Barton E.D., M.L. Argote, J. Brown, M. Kosro, M. Lavin, J.M. Robles, R.L. Smith, A. Trasvina, H.S. Velez (1993). Supersquirt: dynamics of the Gulf of Tehuantepec, MEXICO. Oceanography 6 , 1, 23-30.

Bobier C., E.H. Boumaggard, J. Gayet, E. Aguayo-Camargo, H. Sandoval (1993), Analyse morphostructurale de la marge active du Golfe de Tehuantepec (Mexique- Pacifique) : interprétation géologique et évaluation des composantes horizontale et verticale des mouvements récents. Bull. Inst. Géol. Bassin d'Aquitaine, Bordeaux, 53-54, 121-139.

Bouma A.H. (1962). Sedimentology of some Flysch Deposits. Elsevier, Amsterdam, 168 p.

Boumaggard E.H. (1994). Étude des environnements structuraux et sédimentaires des aires de phosphatogenèse associées au courant d'upwelling sur la marge du Golfe de Tehuantepec (Mexique Pacifique Oriental). Thèse, Université de Bordeaux-I, $\mathrm{n}^{\circ} 1083,311 \mathrm{p}$.

Boumaggard E.H., C. Bobier, J. Gayet, E. Aguayo-Camargo, H. Sandoval (1993). Apport de l'analyse morphostructurale dans la connaissance de la physiographie du Golfe de Tehuantepec (MexiquePacifique). Bull. Inst. Géol. Bassin d'Aquitaine 53-54, 109-120.

Caralp M.H. (1989). Abundance of Bulimina exilis and Melonis barleeanum: relationship to the quality of marine organic matter. Geo-Mar. Lett. 37-43. 
Carfantan J.C. (1986). Du système Cordillerain Nord-Américain au domaine Caraibes. Etude géologique du Mexique méridional. Thèse d'Etat, vol. I et II, Univ. Savoie.

Carranza-Edwards A. (1980). Ambientes sedimentarios recientes de la Llanura Sur del Isthmo de Tehuantepec. An. Inst. Cienc. del Mar y Limnol., Univ. Nal. Autón. México, 7, 2, 13-66.

Carranza-Edwards A. (1986). Estudio sedimentologico de playas del estado de Chiapas México. An. Inst. Cienc. del Mar y Limnol., Univ. Nal. Autón. México, 13, 1, 331-344.

Carranza-Edwards A. (1989). Segundo Informe del Proyecto "Investigaciones Geologicas y Quimicas de Recursos Minerales Marinos Mexicanos". Clave CONACYT: P221 CCON880I4 B, México, $42 \mathrm{p}$.

Carranza-Edwards A., H.L. Rosales, R.E. Ruiz, P.S. Santiago (1989). Investigations of phosphorite deposits in the Gulf of Tehuantepec, Mexico. Marine Mining 11 p.

Cirac P., M. Frappa, E. Jaaidi (1993). Evolution morpho-structurale récente de la plate-forme continentale ouest-rifaine (Maroc nordatlantique). Oceanologica Acta 16, 1, 1-9.

Clarke A.J. (1988). Inertial wind path and sea surface temperature patterns near the gulf of Tehuantepec and gulf of Papagayo. $J$. Geophys. Res. 93, 15491-15501.

Correa 1. (1996). Le littoral pacifique colombien: interdépendance des agents morphostructuraux et hydrodynamiques. Thèse Univ. Bordeaux-I, n ${ }^{\circ} 1538,178 \mathrm{p}$.

Cromwell J.E. (1975). Processes, sediments and history of Laguna Superior, Oaxaca, Mexico. Ph. D. Dissertation Scripps, University of California, San Diego, 143 p.

Curray J.R. (1964). Trangressions and regressions. Papers in Marine Geology, Ed. Mc Millan, New York, p 175-203.

Delgado-Argote L.A., E.A. Carballido-Sanchez (1991). Análisis tectonico del sistema transgresivo neogénico Macuspana, Tabasco y Puerto Angel, Oaxaca. Univ. Nal. Autón. México. Inst. Geología, Revista, 9, $n^{\circ}$ 1, 21-32.0.

Dengo G. (1983). Mid America: tectonic setting for the Pacific IIlargin from Southern Mexico to Northwestern Colombia. In: E.M Nairn Alan, F.G. Stelhi. The Ocean Bassins and Margins, Vol. 7A, The Pacific Ocean, 123-174.

Duplessy J.C., G. Delibrias, J.L. Turon, C. Pujol, J. Duprat (1970). Deglacial warming of the North-Eastern Atlantic Ocean, correlation with the paeoclimatic evolution of the European Continent. Paleogeog., Paleoclimatol., Paleoecol. 35, 121-144.

Gayet J., J.C. Faugères, E. Gonthier (1990). La sédimentation quaternaire récente dans le détroit de Makassar, Oceanologica Acta, Vol. Sp. 10, 307-320.

Heubeck Ch., P. Mann (1991). Geologic evaluation of plate kinematic models for the North American-Caribbean plate boundary zone. Tectonophysics 191, 1/2, 1-27.

Hurd W.E. (1929). Northern of the Gulf of Tchuantepec. Monthly Waether Rev. 57, 5, 192-194.

Lavin M.F., J.M. Robles, M.L. Argote, E.D. Barton, R. Smith, J. Brown, M. Kosro, A. Trasvin, H.S. Velez, J. Garcia (1992). Fisica del Golfo de Tehuantepec. Ciencia y Desarrollo, México, 18, $103,97-107$.
Le Ribault L. (1975). L'exoscopie: Méthodes et Applications. Notes et Mémoires Compagnie Française des Pétroles, Paris 12, $231 \mathrm{p}$

Mac Cave I.N. (1972). Transport and escape of fine grained sediment form shelf areas, In: Shelf sediment transport, process and pattern, Swift, Duane and Pilkey Ed. Dowden, Hutchinson and Ross, 225-248.

McCreary J.P., S. Lee Hyong, D.B. Enfield (1989). The response of the coastal ocean to strong oflshore winds: with application to circulation in the Gulfs of Tehuantepec and Papagayo. J. Mar. Res. 47, 1, 81-109.

Molina-Cruz A. (1984). Radiolaria as indicators of upwelling process: the peruvian connection. Mar. Micropaleontology $9,53-75$.

Molina-Cruz A. (1988). Late Quaternary oceanography of the mouth of the Gulf of California: the policystine connection. Paleoceanography 3, 4, 447-459.

Molina-Cruz A., M. Martinez-Lopez (1994). Oceanography of the Gulf of Tehuantepec, Mexico, indicated by radiolaria remains. Fourth International Palengeog., Polenclimatol, Palenerol. 110, 179-195.

Morales-Garza E. (1990). Estudio de sedimentos fosfatados en el Golfo de Tehuantepec, Mexico. Tesis Maestro en Ciencias del Mar. UNAM, MEXICO, $113 \mathrm{p}$

Perez-Cruz L.L., M.L. Machain (1990). Benthic foraminifera of the oxygen minimum zone, continental shelf of Gulf of Tehuantepec, México. J. Foram. Res. 20, 4, 312-315.

Pichon J.J., L.D. Labeyrie, G. Bareille, M. Labracherie, J. Duprat, J. Jousel (1992). Surface water temperature changes in the high latitudes of the Southern hemisphere over the last Glacial-Interglacial cycle. Paleoceanology 6, 431-460.

Pirazzoli P.A. (1991). World atlas of Holocene sea-level changes. Oceanography Series, Elsevier, Amsterdam, 58, 238 p.

Ramp S.R., P.F. Jessen, K.H. Brink, P.P. Niiler, F.L. Daggett, J.S. Best (1991). The physical structure of cold filaments near Point Arena, California during June 1987. J. Geophys. Res. 96, 14859-14883.

Roden G.I. (1961). On the wind-driven circulation in the Guif of Tehuantepec and its effect upon surface temperatures. Geof. Int. 1, 55-72.

Sanchez-Barreda L.A. (1981). Geologic evolution of the continental margin of the Gulf of Tehuantepec in Southeastern Mexico. Disertation doctoral, Univ, of Texas at Austin, $191 \mathrm{p}$.

Sautter L.R., C. Sancetta (1992). Seasonal associations of phytoplancton and planktonic foraminifera in an upwelling region and their contribution to the seafloor. Mar. Micropaleontology 18 , 263-278.

Stephan J.-F., B. Mercier de Lepinay, E. Calais, M. Tardy, Ch. Beck, J.C. Carfantan, J.L. Olivet, J.M. Vila, Ph. Bouysse, A. Mauffret, J. Bourgois, J.M. Thery, J. Tournon, R. Blanchet, J. Dercourt (1990). Paleogeodynamic maps of the Caribbean: 14 steps from Lias to Present. Bull. Soc. Géol. de France, Bème série, tome VI, No. 6, p. 921-933

Stow D.A.V., G. Shanmugan (1980). Sequence of structures in finegrained turbidites : comparison of recent deep-sea and ancient flysch sediments. Sed. Geol. 25, 23-42.

Stumpf H.P. (1975). Satellite detection of upwelling in the Gulf of Tehuantepec, Mexico. J. Phys. Oceanogr. 5, 383-388. 